Pacific Journal of Mathematics

DEPENDENCE OF DIFFERENTIAL EQUATIONS UPON
PARAMETERS IN THEIR STOKES' MULTIPLIERS 


\title{
DEPENDENCE OF DIFFERENTIAL EQUATIONS UPON PARAMETERS IN THEIR STOKES' MULTIPLIERS
}

\author{
WERNER BALSER
}

Given a system of meromorphic differential equations, an important problem which has attracted increasing attention in recent years is the computation of the Stokes' multipliers. Only in few special cases one can explicitly compute these matrices in terms of known higher transcendental functions in the data of the equation. In general, the non-trivial entries in the Stokes' multipliers appear to be "new" transcendental functions in the data of the equation whose analytic resp. singular behavior should be made as clear as possible-for example, for questions of stability of numerical computations, information on the nature of singularities will certainly be of importance. For the so-called hypergeometric system, fixing the formal invariants, the Stokes' multipliers are entire functions in the rest of the data. Generally, an analogous result on the analytic dependence of Stokes' multipliers for a family of "iso-formal" equations has been obtained by Babbitt and Varadarajan, using more powerful methods.

Roughly speaking, this paper is devoted to a question converse to the one above: Prescribing a Stokes' phenomenon, can one construct a family of equations analytic in the Stokes' multipliers? In principle, this problem is solved positively by results of Birkhoff and, more elegantly, Sibuya on the freedom of the Stokes' multipliers. However, in light of the Birkhoff-Turrittin Reduction Theorem, one should better ask the following (harder) question: Can we construct equations whose coefficient matrix is a polynomial in the independent variable and depends analytically on the Stokes' multipliers? In case of dimension $n=2$, one can see that the entries in such a polynomial equation are multi-valued meromorphic functions of the (two) parameters in the multipliers. In the present paper, this is shown to be true in general. Moreover, we will explicitly find the branch points of these functions and show how to calculate their power series expansion about points of analyticity. In contrast to the situation of $n=2$, we do not know the location and order of their poles.

In order to see how the above question may be phrased in a precise and handy form, suppose that a linear system of differential equations in the complex variable $z$

$$
z x^{\prime}=P(z, u) x
$$

is given, where $P(z, u)$ is a matrix polynomial in $z$ and depends analytically upon a parameter $u$, for $u \in G$, say. Furthermore, 
assume that for every such $u,(0.1)$ admits a formal fundamental solution

$$
H(z, u)=T(z, u) G(z),
$$

where $T(z, u)$ is a formal power series in $z^{-1}$ beginning with the identity matrix $I$, and the remaining coefficients being analytic in $u$; and $G(z)$ is the formal Birkhoff invariant in the sense of [6] and does not depend upon $u$. To every such $u$ and every integer $\nu$, there exists a unique normal solution [6] denoted by $X_{\nu}(z, u)$, so that

$$
X_{\nu}(z, u) \cong H(z, u) \text { as } z \rightarrow \infty \text { in } S_{\nu}
$$

(where $S_{\nu}$ is the $\nu$ th normal sector), and so that the Stokes' multipliers (normalized connection matrices) $V_{\nu}(u)=X_{\nu}^{-1}(z, u) X_{\nu-1}(z, u)$ have a certain prescribed form (which can be made completely explicit in terms of $G(z)$ and hence does not depend upon $u$; see [6] for details). In addition to the above, assume for the moment that the normal solutions (for fixed, but arbitrary $z$ ) and the Stokes' multipliers also are analytic in $u$, for $u \in G$.

If $e^{2 \pi i L}$ denotes the formal monodromy factor, defined by $G\left(z e^{2 \pi i}\right)$ $=G(z) e^{2 \pi i L}$, if $e^{2 \pi i M_{\nu}(u)}$ is the monodromy factor for $X_{\nu}(z, u)$, i.e.

$$
X_{\nu}\left(z e^{2 \pi i}, u\right)=X_{\nu}(z, u) e^{2 \pi i M_{\nu}(u)}
$$

and if $m$ is such that the sectors $S_{1}, \ldots, S_{m}$ cover the complex plane (i.e. on the Riemann surface of the Logarithm, the sector $S_{\nu+m}$ is directly above $S_{\nu}$ ), then the following identities hold (see [6]):

$$
\begin{gathered}
X_{\nu+m}(z, u)=X_{\nu}\left(z e^{-2 \pi i}, u\right) e^{2 \pi i L}, \\
V_{\nu+m}(u)=e^{-2 \pi i L} V_{\nu}(u) e^{2 \pi i L}, \\
e^{2 \pi i M_{\nu}(u)}=e^{2 \pi i L} V_{\nu+m}(u) \cdots V_{\nu+1}(u),
\end{gathered}
$$

for every $u$ and every $z$ (on the Riemann surface of the logarithm), and arbitrary $\nu$.

Suppose that, without loss in generality, the region $G$ contains the origin, and let $P(z), H(z)$, etc., denote the values of $P(z, u)$, $H(z, u)$, etc., for $u=0$. Define

$$
F_{\nu}(z, u)=X_{\nu}(z, u) X_{\nu}^{-1}(z)
$$

then according to (0.3) and the fact that $G(z)$ is independent of $u$, each $F_{\nu}(z, u)$ admits an asymptotic power series expansion in $z^{-1}$ with $I$ as constant term, as $z \rightarrow \infty$ in $S_{\nu}$, and due to $(0.1)$, we have

$$
z F_{\nu}^{\prime}(z, u)=P(z, u) F_{\nu}(z, u)-F_{\nu}(z, u) P(z) .
$$


Moreover, one easily shows

$$
F_{\nu-1}(z, u)=F_{\nu}(z, u)\left(I+X_{\nu}(z) W_{\nu}(u) X_{\nu}^{-1}(z)\right),
$$

where

$$
I+W_{\nu}(u)=V_{\nu}(u) V_{\nu}^{-1}, \quad \text { hence } W_{\nu}(0)=0
$$

and

$$
X_{\nu}(z) W_{\nu}(u) X_{\nu}^{-1}(z) \cong 0 \text { as } z \rightarrow \infty \text { in } S_{\nu-1} \cap S_{\nu} .
$$

The question we are going to investigate is as follows: Given $P(z)$, $G(z)$ and $V_{\nu}(u)$, the latter being analytic for $u \in G$ (for every $\nu$ ), do there exist $P(z, u)$ and $X_{\nu}(z, u)$ (or equivalently, $P(z, u)$ and $F_{\nu}(z, u)$ ), analytic in $u$ for every fixed $z$ and $\nu$, such that the above identities hold? To simplify notations, we will restrict to Stokes' multipliers $V_{\nu}(u)$ which have a very special $u$-dependence (as is explained below, this is sufficient for answering the general question): For an arbitrarily selected integer $\nu_{0}$, let

$$
\begin{aligned}
W_{\nu}(u) & \equiv 0\left(\text { hence } V_{u}(u) \equiv V_{\nu}\right) \text { for } \nu=\nu_{0}+1, \ldots, \nu_{0}+m-1, \\
W_{\nu_{0}} & =u W
\end{aligned}
$$

for some constant matrix $W$, which according to (0.11) satisfies

$$
\begin{gathered}
X(z) W X^{-1}(z) \cong 0 \quad \text { as } z \rightarrow \infty \text { in } S, \\
S=S_{\nu_{0}-1} \cap S_{\nu_{0}}, \quad X(z)=X_{\nu_{0}}(z) .
\end{gathered}
$$

The remaining $W_{\nu}(u)$ (i.e. $V_{\nu}(u)$ ) then may be defined according to (0.5). If $F_{\nu}(z, u)$ as above exist, and if $F(z, u)=F_{\nu_{0}}(z, u)$, then

$$
F_{\nu}(z, u) \equiv F(z, u), \quad \nu=\nu_{0}+1, \ldots, \nu_{0}+m-1,
$$

and (using (0.6))

$$
F\left(z e^{2 \pi i}, u\right)=F(z, u)\left(I+u X(z) W X^{-1}(z)\right) .
$$

As the main result of this paper, we show that given $P(z)$ satisfying an "eigenvalue assumption" (see $\S 1$ ), and a matrix $W$ satisfying (0.12) (in some sector $S=S(\alpha, \beta)$, with some fundamental solution $X(z)$ of $\left.z x^{\prime}=P(z) x\right)$, there exist matrices $F(z, u)$ and $P(z, u)$ which are analytic in $u$, for $|u|$ sufficiently small, and so that $P(z, u)$ is a polynomial in $z, F(z, u)$ has a formal power series expansion in $z^{-1}$ with constant term $I$, as $z \rightarrow \infty$ in the sector $S(\alpha, \beta+2 \pi)$, and satisfies $(0.13)$ and the differential equation $z F^{\prime}(z, u)=P(z, u) F(z, u)-F(z, u) P(z)$. We do so by recursively 
defining the coefficients in the power series expansions of $P(z, u)$ and $F(z, u)(\S 2)$, and through estimates in $\S 3$ we show their convergence for sufficiently small $|u|$ (Theorem 1 ).

Obviously, the matrices $W$ satisfying $(0.12)$ form a vector space $E$ whose dimension only depends upon $G(z)$ (since $(0.12)$ is equivalent to $G(z) W G^{-1}(z) \cong 0$ in $\left.S\right)$ and can be explicitly given [6]. Moreover, from the estimates in $\S 3$ one may easily see that the matrices $W$ fo: which the power series expansions of $P(z, u)$ and $F(z, u)$ converge for $|u| \leq 1$ form a neighborhood of the origin. Moreover, the efficients in these power series $(\S 2)$ are polynomials in several variables $v_{1}, \ldots, v_{q}$, if we select an arbitrary basis of $E$ and identify $E$ with $\mathbb{C}^{q}$ for suitable $q$. Hence we conclude from the uniform convergence of the power series (in $u$ ) that $P(z, 1)$ and $F(z, 1)$ are analytic in the variables $v_{1}, \ldots, v_{q}$ in a suitable polydisc about the origin.

Applying this result to the original question, we have shown that for a given equation $z x^{\prime}=P(z) x$, with a polynomial $P(z)$ that satisfies an eigenvalue assumption, if $G(z), V_{1}, \ldots, V_{m}$ are its Birkhoff invariants (corresponding to an arbitrarily selected formal solution of the form $\left.H(z)=T(z) G(z), T(z)=I+T_{1} z^{-1}+\cdots\right)$, then $P(z)$ depends analytically upon the entries of any one of the Stokes' multipliers $V_{\nu}, 1 \leq \nu \leq m$. With some extra notational efforts, one may see that the same techniques could be used to show that $P(z)$ is, in fact, analytic in all the parameters in $V_{1}, \ldots, V_{m}$ say, in a suitably small polydisc (observe that the parameter space of the normalized connection matrices is isomorphic to $\mathbb{C}^{s}$, with suitable $s$ depending only upon $G(z))$.

In the situation considered in $\S \S 1$ through 3 , we show in $\S 4$ that the functions $P(z, u)$ and $F(z, u)$ are meromorphic in $u$ along every curve provided some polynomial $\rho_{u}(\mu)$ can (as a function of $u$ ) be analytically continued along the curve and satisfies the "eigenvalue assumption" (Theorem 2). Points at which the eigenvalue assumption is violated are (in general) branch points of $P(z, u)$ and $F(z, u)$. From arguments as the ones used above, the proof of Theorem 2 implies that, given $G(z), V_{1}, \ldots, V_{m}$, the polynomial $P(z)$ is a meromorphic function in all the parameters in $V_{1}, \ldots, V_{m}$. Thus in principle, one may construct $P(z)$ having prescribed Birkhoff invariants $G(z), V_{1}, \ldots, V_{m}$ by starting, say, at $V_{1}=\cdots=V_{m}=I$ (i.e. at the origin of the parameter space, where the corresponding $P(z)$ is equal to $z G^{\prime}(z) G^{-1}(z)$ ) and analytically continuing $P(z)$ along a 
curve in the parameter space of $V_{1}, \ldots, V_{m}$ which avoids possible branch points. Due to the uniqueness of polynomial equations with prescribed Birkhoff invariants and prescribed eigenvalues of the monodromy matrix, it may be seen that in case a $P(z)$ having invariants $G(z), V_{1}, \ldots, V_{m}$ and satisfying the eigenvalue assumption exists, it can be constructed by such a continuation process.

1. Polynomial equations as functions of the Stokes' multipliers. Throughout this paper, let $P(z)$ denote an arbitrarily fixed polynomial matrix

$$
P(z)=\sum_{j=0}^{r} P_{j} z^{r-j}, \quad r \geq 1,
$$

where $P_{0}, \ldots, P_{r}$ are $n \times n$ constant matrices $(n \geq 2)$. Let $\rho(\mu)=$ $\sum_{0}^{n} \rho_{j} \mu^{j}=\operatorname{det}\left(P_{r}-\mu I\right)$ be the characteristic polynomial of $P_{r}$, and $\mu_{1}, \ldots, \mu_{n}$ denote the roots of $\rho(\mu)$, repeated according to their multiplicity. We will say that $P(z)$, or sometimes that a polynomial $\rho(\mu)$, satisfies the eigenvalue assumption, if no two roots of $\rho(\mu)$ have a non-zero integer difference.

Let $X(z)$ be an arbitrarily fixed fundamental solution of

$$
z x^{\prime}=P(z) x
$$

and consider a (likewise fixed) sector

$$
S=S(\alpha, \beta)=\{z \neq 0 \mid \alpha<\arg z<\beta\}, \quad \alpha<\beta
$$

(here and throughout, the variable $z$ is considered on the Riemann surface of the logarithm, so that $\beta-\alpha$ might be larger than $2 \pi$, but typically $\beta-\alpha$ is small). A constant matrix $W$ is said to be admissible (with respect to $S$ and $X(z)$ ) iff

$$
X(z) W X^{-1}(z) \cong 0 \text { as } z \rightarrow \infty \text { in } S .
$$

It follows from the asymptotic theory of meromorphic equations [6] that for sufficiently small positive $\beta-\alpha$ there exist $W \neq 0$ which are admissible, except for the (trivial) case where $z=\infty$ is an almost regular singular point of (1.2) (in the sense of [2]). Obviously, the set of admissible $W$ is a matrix algebra. The structure of this algebra and its dependence upon $S$ and $X(z)$ can be made completely explicit (see [6]), but there is no need to do this here.

For an admissible $W$ and a complex parameter $u$, suppose that we find $P(z, u)$, a polynomial matrix in $z$, and $F(z, u)$, analytic in $z$ 
(on the Riemann surface of the logarithm), such that

(1.4) $F(z, u) \cong I+\sum_{k=1}^{\infty} F_{k}(u) z^{-k}$ as $z \rightarrow \infty$ in $\widehat{S}=S(\alpha, \beta+2 \pi)$,

$$
\begin{gathered}
F\left(z e^{2 \pi i}, u\right)=F(z, u)\left(I+u X(z) W X^{-1}(z)\right) \\
z F^{\prime}(z, u)=P(z, u) F(z, u)-F(z, u) P(z)
\end{gathered}
$$

(here and throughout, derivatives will always be with respect to $z$ ). Then (1.6), (1.4) imply

$$
P(z, u)=\sum_{j=0}^{r} z^{r-j} P_{j}(u), \quad P_{0}(u) \equiv P_{0},
$$

and $X(z, u)=F(z, u) X(z)$ is a fundamental solution of

$$
z y^{\prime}=P(z, u) y,
$$

which according to (1.5) satisfies

$$
X\left(z e^{2 \pi i}, u\right)=X(z, u)(I+u W) e^{2 \pi i M}
$$

with

$$
e^{2 \pi i M}=X^{-1}(z) X\left(z e^{2 \pi i}\right) .
$$

If $\rho_{u}(\mu)=\sum \rho_{k}(u) \mu^{k}$ denotes the characteristic polynomial of $P_{r}(u)$, and $\mu_{1}(u), \ldots, \mu_{n}(u)$ its roots, then we conclude from the theory of singularities of first kind that $x_{j}(u)=e^{2 \pi i \mu_{j}(u)}(1 \leq j \leq n)$ are the roots of

$$
\tau_{u}(x)=\operatorname{det}\left\{(I+u W) e^{2 \pi i M}-x I\right\}=\sum_{j=0}^{n} \tau_{k}(u) x^{k} .
$$

Obviously, $\tau_{n}(u) \equiv(-1)^{n}, \quad \tau_{0}(u) \equiv \tau_{0}=\operatorname{det} e^{2 \pi i M}$ (observe that (1.3) implies $\left.\operatorname{det}(I+u W)=\operatorname{det}\left(I+u X(z) W X^{-1}(z)\right) \equiv 1\right)$, and the other $\tau_{k}(u)$ are polynomials in $u$. Moreover, from Abel's formula,

$$
\operatorname{det} X(z, u)=c_{1} z^{\mu(u)} e^{z p(z, u)}=c_{2} f(z, u) z^{\mu_{0}} e^{z p(z)},
$$

with $c_{1}, c_{2} \neq 0$ and

$$
\begin{aligned}
f(z, u) & =\operatorname{det} F(z, u) \cong 1+f_{1}(u) z^{-1}+\cdots \quad \text { as } z \rightarrow \infty \text { in } \hat{S}, \\
\mu(u) & =\operatorname{tr} P_{r}(u), \quad \mu_{0}=\operatorname{tr} P_{r},
\end{aligned}
$$


and polynomials $p(z, u)$ and $p(z)$. This implies $p(z, u) \equiv p(z)$, $\mu(u) \equiv \mu_{0}, f(z, u) \equiv 1, c_{1}=c_{2}$; hence in particular

$$
\sum_{k=1}^{n} \mu_{k}(u) \equiv \mu_{0}=\sum_{k=1}^{n} \mu_{k}, \quad \text { i.e. } \rho_{n-1}(u) \equiv \rho_{n-1} \text {. }
$$

Given $u$, a polynomial $\rho_{u}(\mu)$ with roots $\mu_{j}(u), 1 \leq j \leq n$, will be called admissible iff (1.8) holds and $e^{2 \pi i \mu_{j}(u)}$ are the eigenvalues of $(I+u W) e^{2 \pi i M}$.

LEMMA 1. Let $P(z), X(z)$, and $W$ be as above, and $u$ a complex number. If $\rho_{u}(\mu)$ is an admissible polynomial satisfying the eigenvalue assumption, then there is at most one pair $P(z, u)$ and $F(z, u)$ as above, so that $\rho_{u}(\mu)$ is the characteristic polynomial of $P_{r}(u)$.

Proof. If $\widetilde{P}(z, u)$ and $\widetilde{F}(z, u)$ also have the required properties, then $T(z)=\widetilde{F}(z, u) F^{-1}(z, u)$ is single-valued and has an asymptotic expansion in a full neighborhood of $z=\infty$, with constant term $I$. Hence $T(z)$ is analytic at $\infty$ with $T(\infty)=I$, i.e. $T(z)$ is a Birkhoff transformation. Moreover,

$$
z T^{\prime}(z)=\widetilde{P}(z, u) T(z)-T(z) P(z, u) ;
$$

hence $P(z, u)$ and $\widetilde{P}(z, u)$ are Birkhoff-equivalent standard equations (in the sense of [6]), and since $P_{r}(u)$ and $\widetilde{P}_{r}(u)$ have the same characteristic polynomial satisfying the eigenvalue assumption, we conclude from [6] that $T(z) \equiv I$. This completes the proof.

2. Power series expansions about the origin. We now show the existence of two sequences of matrices which will turn out to be the coefficients of the power series expansions, near $u=0$, of $P(z, u)$, resp. $F(z, u)$.

Proposition 1. With $P(z), X(z)$, and $W$ as above, there exist uniquely defined matrices $Q(z, p), T(z, p)$, holomorphic in $z$ (on the Riemann surface of $\log z)(p \geq 0)$, such that the following holds:

$$
\begin{gathered}
T(z, 0) \equiv I, \quad Q(z, 0) \equiv 0 \\
T(z, p) \cong \sum_{k=1}^{\infty} z^{-k} T_{k}(p) \text { as } z \rightarrow \infty \text { in } \widehat{S} \quad(p \geq 1),
\end{gathered}
$$

$$
Q(z, p)=\sum_{j=1}^{r} Q_{j}(p) z^{r-j} \quad(p \geq 1)
$$


(2.4) $T\left(z e^{2 \pi i}, p\right)=T(z, p)+T(z, p-1) X(z) W X^{-1}(z)$

$$
\begin{aligned}
z T^{\prime}(z, p)= & P(z) T(z, p)-T(z, p) P(z) \\
& +\sum_{q=0}^{p-1} Q(z, p-q) T(z, q) \quad(p \geq 1)
\end{aligned}
$$

Proof. Suppose that $Q(z, p), T(z, p)$ exist as stated. Then (2.5) implies inductively that $T(z, p)$ has a regular point at $z=0$; hence for $y \in(\alpha, \beta)$, the integral

$$
\int_{0}^{\infty(\gamma)} \frac{\zeta^{k-1}}{\zeta-z} X(\zeta) W X^{-1}(\zeta) d \zeta \quad(\arg z \not \equiv \bmod 2 \pi)
$$

exists for sufficiently large natural $k$ (observe (1.3)). From (2.4) it follows (for $R>|z|, \gamma<\arg z<\gamma+2 \pi$ ) with help of Cauchy's formula:

$$
\begin{aligned}
-\int_{0}^{R e^{i \nu}} & \frac{\zeta^{k-1}}{\zeta-z} T(\zeta, p-1) X(\zeta) W X^{-1}(\zeta) d \zeta \\
\quad= & -\int_{0}^{R e^{i(\gamma+2 \pi)}} \frac{\zeta^{k-1}}{\zeta-z} T(\zeta, p) d \zeta+\int_{0}^{R e^{i \gamma}} \frac{\zeta^{k-1}}{\zeta-z} T(\zeta, p) d \zeta \\
& =2 \pi i z^{k-1} T(z, p)-\int_{R e^{i \gamma}}^{R e^{i(\gamma+2 \pi)}} \frac{\zeta^{k-1}}{\zeta-z} T(\zeta, p) d \zeta \\
& =2 \pi i z^{k-1}\left(T(z, p)-\sum_{j=1}^{k-1} T_{j}(p) z^{-j}\right) \\
& -\int_{R e^{i \gamma}}^{R e^{i(\gamma+2 \pi)}} \frac{\zeta^{k-1}}{\zeta-z}\left(T(\zeta, p)-\sum_{j=1}^{k-1} T_{j}(p) \zeta^{-j}\right) d \zeta \quad(p \geq 1),
\end{aligned}
$$

(with the last two integrals taken along the circle $|\zeta|=R$ ).

The last integral tends to zero as $R \rightarrow \infty$ (due to (2.2)), hence

(2.6) $T(z, p)=\sum_{j=1}^{k-1} T_{j}(p) z^{-j}$

$$
-\frac{z^{1-k}}{2 \pi i} \int_{0}^{\infty(\gamma)} \frac{\zeta^{k-1}}{\zeta-z} T(\zeta, p-1) X(\zeta) W X^{-1}(\zeta) d \zeta,
$$


for $\alpha<\gamma<\beta, \gamma<\arg z<\gamma+2 \pi$, and sufficiently large natural $k$. Replacing $k$ by $k+1$ in (2.6) and taking the difference of the resulting and the original formulas, we find

$$
T_{k}(p)=\frac{1}{2 \pi i} \int_{0}^{\infty(\gamma)} \zeta^{k-1} T(\zeta, p-1) X(\zeta) W X^{-1}(\zeta) d \zeta,
$$

for sufficiently large $k$ (and $\gamma$ as above). From (2.5), replacing $T(z, p)$ by its formal expansion and equating coefficients, we obtain

(2.8) $T_{k-r}(p)\left(P_{r}-(k-r) I\right)-P_{r} T_{k-r}(p)$

$$
\begin{aligned}
= & \sum_{j=0}^{r-1}\left(P_{j} T_{k-j}(p)-T_{k-j}(p) P_{j}\right) \\
& +\sum_{q=1}^{p-1} \sum_{j=1}^{r} Q_{j}(p-q) T_{k-j}(q), \quad k \geq r+1, p \geq 1,
\end{aligned}
$$

and

$$
\text { (2.9) } \begin{aligned}
Q_{k}(p)= & -\sum_{j=0}^{k-1}\left(P_{j} T_{k-j}(p)-T_{k-j}(p) P_{j}\right) \\
& -\sum_{q=1}^{p-1} \sum_{j=1}^{k-1} Q_{j}(p-q) T_{k-j}(q), \quad 1 \leq k \leq r, p \geq 1 .
\end{aligned}
$$

Now assume $T(z, q), Q(z, q)$, for $1 \leq q \leq p-1$, given as desired. Then (2.7) determines $T_{k}(p)$ for sufficiently large $k$, and (2.7), together with (2.5) for $p-1$ in place of $p$, can be seen to imply (2.8) for sufficiently large $k$. According to the eigenvalue assumption for $P(z),(2.8)$ is a system of linear equations in the components of $T_{k-r}(p)$ which has a unique solution for $k \geq r+1$; hence $T_{k}(p)$ is uniquely defined for $k \geq 1$. From (2.9) we then obtain $Q(z, p)$, and from (2.6) we have $T(z, p)$. It is now straightforward to check that (2.2), (2.4), and (2.5) hold. This completes the proof.

For a complex parameter $s$, define a sequence of matrices $W(s, p)$ by

$$
e^{2 \pi i(s I+M)} W(s, 1)-W(s, 1) e^{2 \pi i M}=-W e^{2 \pi i M},
$$

and for $p \geq 2$ :

$$
\begin{gathered}
e^{2 \pi i(s I+M)} W(s, p)-W(s, p) e^{2 \pi i M} \\
=-W e^{2 \pi i(s I+M)} W(s, p-1)
\end{gathered}
$$


Obviously, the above equations determine $W(s, p)$ recursively for every $s$ satisfying

$$
s \not \equiv \mu_{j}-\mu_{k} \bmod 1, \quad 1 \leq j, k \leq n ;
$$

hence $W(s, p)(p \geq 1)$ is a meromorphic function in $s$, with possible poles at the points excluded by $(2.12)$.

Proposition 2. Under the assumptions of Proposition 1 , for $p \geq 1$ and $s$ satisfying (2.12), let

$$
\begin{aligned}
T(z, p, s)= & \frac{-1}{2 \pi i} \int_{R^{i \gamma}}^{\infty(\gamma)} \frac{\zeta^{s}}{\zeta-z} T(\zeta, p-1) X(\zeta) W X^{-1}(\zeta) d \zeta \\
& +\sum_{q=0}^{p-1} \frac{1}{2 \pi i} \int_{R e^{i \gamma}}^{R e^{i(\gamma+2 \pi)}} \frac{\zeta^{s}}{\zeta-z} T(\zeta, q) X(\zeta) \\
& \times W(s, p-q) X^{-1}(\zeta) d \zeta,
\end{aligned}
$$

with $R>0, \alpha<\gamma<\beta,|z|>R, \gamma<\arg z<\gamma+2 \pi$ (integrating in the first integral along $\arg \zeta=\gamma$, in the remaining ones along $|\zeta|=R$ ). Then $T(z, p, s)$ (for fixed $s$ ) is holomorphic in $z$ and (for fixed $z$ ) meromorphic in $s$ with possible poles along the points where (2.12) is violated. In particular, $T(z, p, s)$ has removable singularities at points of the form

$$
s=\mu_{j}-\mu_{k}+\mu, \quad 1 \leq j, k \leq n,
$$

with $\mu$ being a non-negative integer, and

$$
T(z, p, 0) \equiv T(z, p) .
$$

Proof. From(2.4), (2.10), (2.11) we conclude

$$
\begin{aligned}
\sum_{q=0}^{p-1}\left(\zeta e^{2 \pi i s}\right) T\left(\zeta e^{2 \pi i}, q\right) X\left(\zeta e^{2 \pi i}\right) W(s, p-q) X^{-1}\left(\zeta e^{2 \pi i}\right) \\
=-\zeta^{s} T(\zeta, p-1) X(\zeta) W X^{-1}\left(\zeta e^{2 \pi i}\right) \\
\quad+\sum_{q=0}^{p-1} \zeta^{s} T(\zeta, q) X(\zeta) W(s, p-q) X^{-1}(\zeta)
\end{aligned}
$$

Therefore, $T(z, p, s)$ does not depend upon the choice of $R$, and if $\operatorname{Re} s$ is sufficiently large, then

$$
T(z, p, s)=\frac{-1}{2 \pi i} \int_{0}^{\infty(\gamma)} \frac{\zeta^{s}}{\zeta-z} T(\zeta, p-1) X(\zeta) W X^{-1}(\zeta) d \zeta .
$$


Consequently, $T(z, p, s)$ has no poles in $s$ for such values of $s$ for which the above integral exists, i.e. for $\operatorname{Re} s>s_{0}$. For arbitrary $s$ with (2.12), let

$$
\begin{aligned}
T_{s}(p)= & \lim _{z \rightarrow \infty} z T(z, p, s-1) \\
= & \frac{1}{2 \pi i} \int_{R e^{i \gamma}}^{\infty(\gamma)} \zeta^{s-1} T(\zeta, p-1) X(\zeta) W X^{-1}(\zeta) d \zeta \\
& -\sum_{q=0}^{p-1} \frac{1}{2 \pi i} \int_{R e^{i \gamma}}^{R e^{i(\gamma+2 \pi)}} \zeta^{s-1} T(\zeta, q) X(\zeta) \\
& \times W(s, p-q) X^{-1}(\zeta) d \zeta,
\end{aligned}
$$

then for $N \geq 0$ (integer)

$$
T(z, p, s)=\sum_{k=1}^{N} z^{-k} T_{s+k}(p)+z^{-N} T(z, p, s+N) .
$$

This shows that the poles of $T(z, p, s)$ are among those of $T_{s+k}(p)$, $k \geq 1$. But from (2.16), by means of integration by parts and (2.5),

$$
\begin{aligned}
T_{s}(p)\left(P_{r}-s I\right)-P_{r} T_{s}(p)= & \sum_{j=0}^{r-1}\left(P_{j} T_{s+r-j}(p)-T_{s+r-j} P_{j}\right) \\
& +\sum_{q=1}^{p-1} \sum_{j=1}^{r} Q_{j}(p-q) T_{s+r-j}(q),
\end{aligned}
$$

which gives the analytic continuation of $T_{s}(p)$ to every point $s$ except $s=\mu_{j}-\mu_{k}-\nu, 1 \leq j, k \leq n$, and $\nu=0,1,2, \ldots$. This completes the proof, in view of $(2.6),(2.7)$.

3. Estimates. For arbitrarily fixed $\delta, 0<\delta<(\beta-\alpha) / 2$, let

$$
\begin{aligned}
& S_{\delta}=\{z|| z \mid \geq \delta, \quad \alpha+\delta \leq \arg z \leq \beta-\delta\}, \\
& \widehat{S}_{\delta}=\{z|| z \mid \geq \delta, \quad \alpha+\delta \leq \arg z \leq \beta+2 \pi-\delta\},
\end{aligned}
$$

(on the Riemann surface of the Logarithm). For $p \geq 0$, define

$$
c_{p}=\max \left\{\|T(z, p)\| \mid z \in \widehat{S}_{\delta}\right\}
$$

$$
c_{p}^{(j)}=\max \left\{\|T(z, p)\| \mid z \in A_{j}\right\}, \quad j=1,2,3,
$$


with

$$
\begin{aligned}
A_{1} & =\{z|\arg z=\alpha+\delta,| z \mid \geq \delta+1\}, \\
A_{2} & =\{z|\arg z=\beta+2 \pi-\delta,| z \mid \geq \delta+1\}, \\
A_{3} & =C \cup B_{1} \cup B_{2}, \\
C & =\{z|| z \mid=\delta, \alpha+\delta \leq \arg z \leq \beta+2 \pi-\delta\}, \\
B_{1} & =\{z|\arg z=\alpha+\delta, \delta \leq| z \mid \leq \delta+1\}, \\
B_{2} & =\{z|\arg z=\beta+2 \pi-\delta, \delta \leq| z \mid \leq \delta+1\}
\end{aligned}
$$

(here and throughout, as norm of a matrix or a vector, we use the maximum modulus of its components). Note that obviously

$$
c_{0}=c_{0}^{(j)}=1, \quad 1 \leq j \leq 3,
$$

and due to (2.2), all the maxima exist. Moreover, the Maximum Modulus Principle implies

$$
c_{p}=\max \left\{c_{p}^{(1)}, c_{p}^{(2)}, c_{p}^{(3)}\right\},
$$

since $A_{1} \cup A_{2} \cup A_{3}$ is the boundary of $\widehat{S}_{\delta}$.

LEMMA 2. With $P(z), X(z)$, and $W$ as above, there exist positive constants $K_{1}, K_{2}$ such that

$$
c_{p} \leq K_{1} \sum_{q=0}^{p-1} K_{2}^{p-q} c_{q}, \quad p \geq 1
$$

Proof. From Proposition 2 and the Maximum Modulus Principle,

$$
\|T(z, p)\| \leq \max \{\|T(z, p, s)\||| s \mid=\rho\},
$$

if we choose $\rho>0$ small enough. From (2.10), (2.11), we find for $|s|=\rho$

$$
\|W(s, p)\| \leq w^{p}, \quad p \geq 1,
$$

if we take $w>0$ large enough. Using (2.13) we find for

$$
R \geq \delta, \quad \alpha+\delta \leq \gamma \leq \beta-\delta, \quad|z|>R, \quad \gamma<\arg z<\gamma+2 \pi:
$$

$$
\|T(z, p)\| \leq \frac{n c_{p-1}}{d_{1}(z) 2 \pi} a_{1} \int_{R}^{\infty} \frac{d x}{x^{2}}+\frac{n^{2} a_{2}}{d_{2}(z)} R \sum_{q=0}^{p-1} c_{q} w^{p-q}
$$


with

$$
\begin{aligned}
& d_{1}(z)=\min \{|z-\zeta||| \zeta \mid \geq R, \quad \arg \zeta=\gamma\}, \\
& d_{2}(z)=\min \{|z-\zeta||| \zeta \mid=R, \gamma \leq \arg \zeta \leq \gamma+2 \pi\}=|z|-R, \\
& a_{1}=\max \left\{\left\|\zeta^{s+2} X(\zeta) W X^{-1}(\zeta)\right\||| s \mid=\rho, \zeta=x e^{i \gamma}, x \geq R\right\}, \\
& a_{2}=\max \left\{\left|\zeta^{s}\right|\|X(\zeta)\|\left\|X^{-1}(\zeta)\right\||| s \mid=\rho, \zeta=R e^{i \varphi}\right. \text {, } \\
& \gamma \leq \varphi \leq \gamma+2 \pi\} \text {. }
\end{aligned}
$$

If we take $|z| \geq \delta+1, \arg z=\alpha+2 \pi+\delta$, then for $R=\delta, \gamma=\beta-\delta$ we have

$$
d_{1}(z) \geq d_{1}>0(\text { independent of } z), \quad d_{2}(z) \geq 1 ;
$$

hence for such $z$, there exist $k_{1}, k_{2}>0$ (independent of $p$ ) so that

$$
\|T(z, p)\| \leq k_{1} \sum_{q=0}^{p-1} k_{2}^{p-q} c_{q}, \quad p \geq 0 .
$$

From (2.4), we find

$$
c_{p}^{(1)} \leq n c_{p-1} k_{0}+\max \{\|T(z, p)\||| z \mid \geq \delta+1, \arg z=\alpha+2 \pi+\delta\},
$$

with

$$
k_{0}=\max \left\{\left\|X(z) W X^{-1}(z)\right\| \mid z \in S_{\delta}\right\},
$$

hence we find $K_{1}, K_{2}>0$ (independent of $p$ ), so that

$$
c_{p}^{(1)} \leq K_{1} \sum_{q=0}^{p-1} K_{2}^{p-q} c_{q}, \quad p \geq 1 .
$$

Analogously, one can show the same estimate for $c_{p}^{(2)}$ (enlarging $K_{1}$, $K_{2}$ if necessary). To estimate $c_{p}^{(3)}$, we observe that the integrals in (2.13) give sense for $|z|<R$ and define an analytic function $\widetilde{T}(z, p, s)$ (even entire, since $R$ is arbitrary). Using Cauchy's integral formula and deforming paths of integration, we find

$$
\begin{aligned}
T(z, p, s)= & \widetilde{T}(z, p, s) \\
& +z^{s} \sum_{q=0}^{p-1} T(z, q) X(z) W(s, p-q) X^{-1}(z)
\end{aligned}
$$

for $0<|z|<R, \gamma<\arg z<\gamma+2 \pi$. Estimating the integral formula for $\widetilde{T}(z, p, s)$ (for $R=\delta+2, \gamma=\alpha+\delta$, and $z \in A_{3}$ ) it is then easy to show that $c_{p}^{(3)}$ can be estimated analogously to $c_{p}^{(1)}, c_{p}^{(2)}$. This then completes the proof. 
Theorem 1. With $P(z), X(z)$, and $W$ as above, there exist $P(z, u), F(z, u)$, analytic in $u$ for every $u$ with $|u|<\rho$ (for suffciently small $\rho>0$, independent of $z$ ) and every fixed $z$, such that (1.4), (1.5), (1.6) hold for every such $z$ and $u$, and

$$
P(z, 0) \equiv P(z), \quad F(z, 0) \equiv I .
$$

Proof. With $T(z, p), Q(z, p)$ as in Proposition 1, define

$$
F(z, u)=\sum_{p=0}^{\infty} u^{p} T(z, p) .
$$

According to Lemma 2, the series $\sum_{p=0}^{\infty} f_{p} u^{p}$, with

$$
f_{0}=1, \quad f_{p}=K_{1} \sum_{q=0}^{p-1} K_{2}^{p-q} f_{q}, \quad p \geq 1,
$$

is a majorant for the above power series, and

$$
\begin{aligned}
f(u) & =\sum_{p=0}^{\infty} f_{p} u^{p}=1+\sum_{p=1}^{\infty} u^{p} K_{1} \sum_{q=0}^{p-1} K_{2}^{p-q} f_{q} \\
& =1+\frac{u K_{1} K_{2}}{1-u K_{2}} f(u)
\end{aligned}
$$

implies

$$
f(u)=\frac{1-u K_{2}}{1-u K_{2}\left(1+K_{1}\right)}
$$

i.e., $f(u)$ is holomorphic for $|u|<\left(K_{2}\left(1+K_{1}\right)\right)^{-1}$. This shows that $F(z, u)$ is analytic in $u$, for $|u|<\left(K_{2}\left(1+K_{1}\right)\right)^{-1}$ and every fixed $z$. Since (2.2) implies (1.4), hence $F(z, u)$ invertible, at least for $|z|$ large enough, we may define

$$
P(z, u)=F^{-1}(z, u)\left[P(z) F(z, u)-z F^{\prime}(z, u)\right],
$$

and find that $P(z, u)$, for fixed $z$, is analytic in $u$, for $u$ as above. Equating like powers of $u$, we find from (2.5)

$$
P(z, u)=P(z)+\sum_{p=1}^{\infty} u^{p} Q(z, p), \quad|u|<\rho ;
$$

hence $P(z, u)$ is a polynomial in $z$, and (1.6) holds. From (2.4) it is now easy to prove (1.6). 
4. The meromorphic nature of $P(z, u)$. For an admissible polynomial $\rho_{u}(\mu)$, there may not exist $P(z, u), F(z, u)$ satisfying (1.4), (1.5), (1.6), so that $P_{r}(u)=P(0, u)$ has characteristic polynomial $\rho_{u}(\mu)$. However, the following is true:

Proposition 3. With $P(z), X(z), W$ as above and a complex number $u$, let $\rho_{u}(\mu)$ be an arbitrary admissible polynomial. Then there exists a polynomial $\widetilde{P}(z)$ (generally of degree larger than $r$ ) with $\widetilde{P}(0)$ having the characteristic polynomial $\rho_{u}(\mu)$, a matrix $T(z)$, analytic on the Riemann surface of the Logarithm, and a diagonal matrix $K$ of integer diagonal entries, with $\operatorname{tr} K=0$, such that

$$
\begin{gathered}
T(z) \cong I+\sum_{k=1}^{\infty} T_{k} z^{-k} \quad \text { as } z \rightarrow \infty \text { in } \widehat{S}, \\
z Y^{\prime}(z)=\widetilde{P}(z) Y(z),
\end{gathered}
$$

with

$$
\begin{gathered}
Y(z)=T(z) z^{K} X(z), \\
Y\left(z e^{2 \pi i}\right)=Y(z)(I+u W) e^{2 \pi i M} .
\end{gathered}
$$

Proof. According to a theorem of Sibuya's [7], formulated in [6], p. 107 , we deduce the existence of $\widetilde{T}(z)$, analytic for $|z|$ sufficiently large (but not as $z=\infty$ ) and having a formal power series expansion with leading term $I$, as $z \rightarrow \infty$ in $\widehat{S}$, so that

$$
\widetilde{T}\left(z e^{2 \pi i}\right)=\widetilde{T}(z)\left(I+u X(z) W X^{-1}(z)\right) .
$$

The matrix

$$
\begin{aligned}
A(z) & =z[\widetilde{T}(z) X(z)]^{\prime}[\widetilde{T}(z) X(z)]^{-1} \\
& =\widetilde{T}(z) P(z) \widetilde{T}^{-1}(z)+z \widetilde{T}^{\prime}(z) \widetilde{T}^{-1}(z)
\end{aligned}
$$

is easily seen to be single-valued and analytic, for $|z|$ large enough, and has a pole of order $\leq r$ at $z=\infty$. The meromorphic equation

$$
z y^{\prime}=A(z) y
$$

is, according to the Birkhoff-Turrittin Reduction Theorem (compare [6]), meromorphically equivalent to a polynomial equation

$$
z x^{\prime}=\widehat{P}(z) x
$$


with $\widehat{P}(0)$ having the characteristic polynomial $\rho_{u}(\mu)$. Hence there exists a matrix $\widehat{T}(z)$, analytic on the Riemann surface of the Logarithm, having a formal meromorphic transformation as its formal expansion, as $z \rightarrow \infty$ in $\widehat{S}$, such that the matrix $\widehat{Y}(z)=\widehat{T}(z) X(z)$ satisfies

$$
\begin{aligned}
z \widehat{Y}^{\prime}(z) & =\widehat{P}(z) \widehat{Y}(z), \\
\widehat{Y}\left(z e^{2 \pi i}\right) & =\widehat{Y}(z)(I+u W) e^{2 \pi i M} .
\end{aligned}
$$

Applying [4], Lemma 2, to the formal expansion of $\widehat{T}(z)$, we find

$$
\widehat{T}(z)=Q(z) T(z) z^{K},
$$

with $K$ and $T(z)$ as in Proposition 3, in particular (4.1), and a polynomial matrix $Q(z)$, with constant non-zero determinant. Defining $Y(z)=Q^{-1}(z) \widehat{Y}(z)$ and $\widetilde{P}(z)$ by $(4.2)$, one easily finds that $\widetilde{P}(z)$ is again a polynomial in $z$, and $\widetilde{P}(0)$ has the same eigenvalues as $\widehat{P}(0)$. Moreover, (4.3) and (4.4) hold. Finally, an application of Abel's formula and (1.8) show that $\operatorname{tr} K=0$. Thus, the proof is completed.

From Theorem 1 we conclude that, for every $u$ with $|u|<\rho$, there is an admissible polynomial $\rho_{u}(\mu)=\sum_{1}^{n} \rho_{k}(u) \mu^{k}$ which is the characteristic polynomial of $P_{r}(u)$, and consequently $\rho_{k}(u)$ is an analytic function for $|u|<\rho, 1 \leq k \leq n$. If we are given a curve along which these functions $\rho_{k}(u)$ may be analytically continued, then the following Theorem 2 shows that $P(z, u)$ and $F(z, u)$ are meromorphic in $u$ along this curve, provided that $\rho_{u}(\mu)$, for every $u$ on the curve, satisfies the eigenvalue assumption. The following remark shows that the eigenvalue assumption is in fact closely related to the analyticity of the $\rho_{k}(u)$.

REMARK 4.1. For $P(z), X(z)$, and $W$ as above, define $\tau_{u}(x)=$ $\sum \tau_{k}(u) x^{k}=\prod\left(x-x_{k}(u)\right)$ as in $\S 1$. Suppose that $\tau_{u}(x)$ is irreducible over the field of rational functions (otherwise, treat every irreducible factor of $\tau_{u}(x)$ separately). Then there is a finite set $A$ so that for $u \notin$ $A$ the roots $x_{1}(u), \ldots, x_{n}(u)$ of $\tau_{u}(x)$ are all distinct (and different from zero). Therefore, one can easily see that

$$
\mu_{j}(u)=\frac{\log x_{j}(u)}{2 \pi i}, \quad 1 \leq j \leq n,
$$

are analytic functions along every curve avoiding $A$ (provided that we decide upon a branch of the Logarithm, which may depend upon $j$, at one end of the curve). Consequently, the $\rho_{k}(u)$ can be analytically 
continued along every curve starting near the origin and avoiding $A$. For $u_{0} \in A$, consider a circle $\mathbf{k}$ around $u_{0}$ (positively oriented) of so small a radius that no other point of $A$ lies inside or on $\mathbf{k}$, and assume that we have analytically continued $\rho_{k}(1 \leq k \leq n)$ to some $u \in \mathbf{k}$. If we analytically continue the algebraic function $x_{j}(u)$ once around $\mathbf{k}$, it may happen that we end up with, say, $x_{\pi(j)}(u)$. The analytic continuation of $\mu_{j}(u)$ (i.e. of $\log x_{j}(u)$ ) may lead to a value $\tilde{\mu}_{j}(u)$ which may or may not coincide with $\mu_{\pi(j)}(u)$ (since we may or may not end up with the preselected branch $\left.\log x_{\pi(j)}(u)\right)$. In any case, there is an integer $k_{j}$ so that

$$
\tilde{\mu}_{j}(u)=\mu_{\pi(j)}(u)+k_{j} .
$$

Since $u \notin A$, we have $x_{1}(u), \ldots, x_{n}(u)$ all distinct; hence $\mu_{1}(u), \ldots$, $\mu_{n}(u)$ are all incongruent modulo one. Therefore, if $k_{j} \neq 0$, then $\tilde{\mu}_{j}(u)$ cannot be a root of $\rho_{u}(\mu)$; hence at least one of the functions $\rho_{j}$ cannot be single-valued. Conversely, if $k_{j}=0$ for every $j=1, \ldots, n$, then the analytic continuation of the $\rho_{k}(u)$ leads to a polynomial having the same roots as $\rho_{u}(\mu)$, and hence $\rho_{k}(u)$ are single-valued and therefore even analytic at $u=u_{0}$. For $u \rightarrow u_{0}$ along an arbitrarily fixed curve, we have $x_{j}(u) \rightarrow x_{j}\left(u_{0}\right) \neq 0$, and hence $\mu_{j}(u) \rightarrow \mu_{j}\left(u_{0}\right)$, with $x_{j}\left(u_{0}\right)$ and $\mu_{j}\left(u_{0}\right)$ independent of the chosen curve. Consequently, $\tilde{\mu}_{j}(u)$ also tends to $\mu_{j}\left(u_{0}\right)$, which implies $\tilde{\mu}_{j}\left(u_{0}\right)=\mu_{\pi(j)}\left(u_{0}\right)+k_{j}(1 \leq j \leq n)$. Since $x_{j}\left(u_{0}\right)=x_{\pi(j)}\left(u_{0}\right)$, we find: if $\rho_{u_{0}}(\mu)=\lim _{u \rightarrow u_{0}} \rho_{u}(\mu)$ (along that fixed curve) satisfies the eigenvalue assumption, then $k_{j}=0(1 \leq j \leq n)$, and hence all $\rho_{k}(u)$ are analytic at $u=u_{0}$. The converse does not hold in general, as one may see from examples (observe that $\pi(j)=j$ may hold for every $j$, which implies analyticity of every $\rho_{k}(u)$ at $u_{0}$, even if the eigenvalue assumption for $\rho_{u_{0}}(\mu)$ fails).

Theorem 2. With $P(z), X(z)$, and $W$ as above, the matrices $P(z, u)$ and $F(z, u)$ (which are analytic in $u$, for small $|u|$, according to Theorem 1) are meromorphic in $u$, for $u$ on an arbitrary curve originating from $u=0$, provided that the functions $\rho_{k}(u)$ can be analytically continued along this curve, so that $\rho_{u}(\mu)=\sum_{1}^{n} \rho_{k}(u) \mu^{k}$ satisfies the eigenvalue assumption for arbitrary $u$ on the curve.

Proof. Let $u(t), 0 \leq t \leq 1$, be a parametrization of some curve as described in Theorem 2 , and let $t_{0}$ be so that $F(z, u)$ and $P(z, u)$ are meromorphic for $u=u(t), 0 \leq t<t_{0}$, (with some $t_{0} \leq 1$ ). Applying Proposition 3 with $u=u\left(t_{0}\right)=u_{0}$, we find $\widetilde{P}(z), T(z)$, 
$K$, and $Y(z)$ as stated there. Applying Theorem 1 with $\widetilde{P}(z), Y(z)$, $W\left(I+u_{0} W\right)^{-1}$, and $\tilde{u}=u-u_{0}$ replacing $P(z), X(z), W$, and $u$, resp. (check that the matrix $\widetilde{W}=W\left(I+u_{0} W\right)^{-1}$ is admissible with respect to $Y(z)$ and $S)$, we obtain $\widetilde{P}(z, \tilde{u}), \widetilde{F}(z, \tilde{u})$, analytic in $\tilde{u}$, for $|\tilde{u}|<\rho$, so that $\widetilde{P}(z, 0)=\widetilde{P}(z), \widetilde{F}(z, 0) \equiv I$, and

$$
\begin{aligned}
\widetilde{F}(z, \tilde{u}) & \cong I+\sum_{k=1}^{\infty} \widetilde{F}_{k}(\tilde{u}) z^{-k} \text { as } z \rightarrow \infty \text { in } \widehat{S}, \\
\widetilde{F}\left(z e^{2 \pi i}, \tilde{u}\right) & =\widetilde{F}(z, \tilde{u})\left(I+\tilde{u} Y(z) \widetilde{W} Y^{-1}(z)\right), \\
z \widetilde{F}^{\prime}(z, \tilde{u}) & =\widetilde{P}(z, \tilde{u}) \widetilde{F}(z, \tilde{u})-\widetilde{F}(z, \tilde{u}) \widetilde{P}(z) .
\end{aligned}
$$

With

$$
\begin{aligned}
\widehat{F}(z, u) & =\widetilde{F}(z, \tilde{u}) T(z), \\
\widehat{X}(z, u) & =\widehat{F}(z, u) z^{K} X(z)=\widetilde{F}(z, \tilde{u}) Y(z), \\
\widehat{P}(z, u) & =\widetilde{P}(z, \tilde{u}),
\end{aligned}
$$

one checks that for $u$ with $\left|u-u_{0}\right|<\rho$

$$
\begin{gathered}
z \widehat{X}^{\prime}(z, u)=\widehat{P}(z, u) \widehat{X}(z, u), \\
\widehat{F}(z, u) \cong I+\sum_{k=1}^{\infty} \widehat{F}_{k}(u) z^{-k} \quad \text { as } z \rightarrow \infty \text { in } \widehat{S}, \\
\widehat{X}\left(z e^{2 \pi i}, u\right)=\widehat{X}(z, u)(I+u W) e^{2 \pi i M} .
\end{gathered}
$$

Moreover, since $\widehat{P}_{r}(u)=\widehat{P}(0, u)$ is analytic for $\left|u-u_{0}\right|<\rho$, and since the characteristic polynomial of $\widehat{P}_{r}\left(u_{0}\right)$ equals $\rho_{u_{0}}(\mu)$, we conclude that for every $u$ with $\left|u-u_{0}\right|<\rho$ the characteristic polynomial of $\widehat{P}_{r}(u)$ equals $\rho_{u}(\mu)$.

For fixed $u,\left|u-u_{0}\right|<\rho$, assume that $P(z, u)$ and $F(z, u)$ satisfying (1.4), (1.5), (1.6) exist, with $\rho_{u}(\mu)$ being the characteristic polynomial of $P_{r}(u)=P(0, u)$. Defining

$$
T(z, u)=F(z, u) z^{-K} \widehat{F}^{-1}(z, u),
$$

we obtain from (4.8), (1.5), (4.7), (1.4) that $T(z, u)$ is single-valued and meromorphic at $z=\infty$. Moreover, we conclude from (4.6) and (1.6) that

$$
z T^{\prime}(z, u)=P(z, u) T(z, u)-T(z, u) \widehat{P}(z, u),
$$

and since $\widehat{P}_{r}(u)$ and $P_{r}(u)$ have the same characteristic polynomial $\rho_{u}(\mu)$ satisfying the eigenvalue assumption, this in turn implies that 
$T(z, u)$ is a polynomial in $z$ with constant, non-zero determinant. From [4], Proposition 1 we conclude (replacing $F(z, u)$ and $\widehat{F}(z, u)$ in (4.9) by their formal expansions) that such a $T(z, u)$ exists iff an explicitly given function in the columns of (finitely many) $\widehat{F}_{k}(u)$ is non-zero; this function obviously is analytic in $u$, for $\left|u-u_{0}\right|<\rho$, and may be denoted by $d(u)$. Since $F(z, u)$, and hence $T(z, u)$, do exist for $u=u(t), t<t_{0}$ we observe that $d(u) \not \equiv 0$. The formal identity

$$
T(z, u)\left(I+\sum_{1}^{\infty} \widehat{F}_{k}(u) z^{k}\right) z^{K}=I+O\left(z^{-1}\right)
$$

is a system of linear equations in the coefficients $T_{k}(u)$ of the polynomial $T(z, u)$, having a unique solution iff $d(u) \neq 0$, hence $T(z, u)$ is a meromorphic function in $u$, for $\left|u-u_{0}\right|<\rho$. Solving (4.9) for $F(z, u)$, and defining $P(z, u)$ by (1.6), this completes the proof.

\section{REFERENCES}

[1] D. G. Babbitt and V. S. Varadarajan, Deformations of nilpotent matrices over rings and reduction of analytic families of meromorphic differential equations, Mem. Amer. Math. Soc., 55 No. 325 (1985).

[2] W. Balser, Convergent power series expansions for the Birkhoff invariants of meromorphic differential equations; Part I: Definition of the coefficient functions, Yokohama Math. J., 32 (1984), 15-29. Part II: A closer study of the coefficients, Yokohama Math. J., 33 (1985), 7-19.

[3] _ Growth estimates for the coefficients of generalized formal solutions, and representation of solutions using Laplace integrals and factorial series, Hirosh. Math. J., 12 (1982), 11-42.

[4] —-, Meromorphic transformation to Birkhoff standard form in dimension three; J. Fac. Sc. Univ. Tokyo, 36 (1989), 233-246.

[5] W. B. Jurkat, D. A. Lutz, and A. Peyerimhoff, Birkhoff invariants and effective calculations for meromorphic linear differential equations; Part I: J. Math. Anal. Appl., 53 (1976), 438-470. Part II: Houston J. Math., 2 (1976), 207-238.

[6] W. B. Jurkat, Meromorphe Differentialgleichungen, Lecture Notes in Math., 637 (1978) (entire volume).

[7] Y. Sibuya, Linear Differential Equations in the Complex Domain; Problems of Analytic Continuation (in Japanese) Kinokuniya, Tokyo.

Received December 14, 1989.

UNIVERSITAET ULM

ObERER ESELSBERG

D-7900 Ulm/DONAU

WEST GERMANY 



\section{PACIFIC JOURNAL OF MATHEMATICS EDITORS}

\author{
V. S. VARAdaraJan \\ (Managing Editor) \\ University of California \\ Los Angeles, CA 90024-1555-05 \\ Herbert Clemens \\ University of Utah \\ Salt Lake City, UT 84112 \\ THOMAS ENRIGHT \\ University of California, San Diego \\ La Jolla, CA 92093
}

R. FINN

Stanford University

Stanford, CA 94305

Hermann FlaschKa

University of Arizona

Tucson, AZ 85721

VAUGHAN F. R. Jones

University of California

Berkeley, CA 94720

STEVEN KerckHofF

Stanford University

Stanford, CA 94305

\section{C. MOORE}

University of California

Berkeley, CA 94720

Martin ScharlemanN

University of California

Santa Barbara, CA 93106

HAROLd STARK

University of California, San Diego

La Jolla, CA 92093
R. ARENS
E. F. BECKENBACH
F. WoLF
K. YoshidA
ASSOCIATE EDITORS

\begin{tabular}{ll}
\multicolumn{1}{c}{$(1906-1982)$} & \multicolumn{1}{c}{$(1904-1989)$} \\
SUPPORTING & INSTITUTIONS \\
UNIVERSITY OF ARIZONA & UNIVERSITY OF OREGON \\
UNIVERSITY OF BRITISH COLUMBIA & UNIVERSITY OF SOUTHERN CALIFORNIA \\
CALIFORNIA INSTITUTE OF TECHNOLOGY & STANFORD UNIVERSITY \\
UNIVERSITY OF CALIFORNIA & UNIVERSITY OF HAWAII \\
MONTANA STATE UNIVERSITY & UNIVERSITY OF TOKYO \\
UNIVERSITY OF NEVADA, RENO & UNIVERSITY OF UTAH \\
NEW MEXICO STATE UNIVERSITY & WASHINGTON STATE UNIVERSITY \\
OREGON STATE UNIVERSITY & UNIVERSITY OF WASHINGTON
\end{tabular}

The Supporting Institutions listed above contribute to the cost of publication of this Journal, but they are not owners or publishers and have no responsibility for its content or policies.

Mathematical papers intended for publication in the Pacific Journal of Mathematics should be in typed form or offset-reproduced (not dittoed), double spaced with large margins. Please do not use built up fractions in the text of the manuscript. However, you may use them in the displayed equations. Underline Greek letters in red, German in green, and script in blue. The first paragraph must be capable of being used separately as a synopsis of the entire paper. In particular it should contain no bibliographic references. Please propose a heading for the odd numbered pages of less than 35 characters. Manuscripts, in triplicate, may be sent to any one of the editors. Please classify according to the 1991 Mathematics Subject Classification scheme which can be found in the December index volumes of Mathematical Reviews. Supply name and address of author to whom proofs should be sent. All other communications should be addressed to the managing editor, or Elaine Barth, University of California, Los Angeles, California 90024-1555-05.

There are page-charges associated with articles appearing in the Pacific Journal of Mathematics. These charges are expected to be paid by the author's University, Government Agency or Company. If the author or authors do not have access to such Institutional support these charges are waived. Single authors will receive 50 free reprints; joint authors will receive a total of 100 free reprints. Additional copies may be obtained at cost in multiples of 50 .

The Pacific Journal of Mathematics (ISSN 0030-8730) is published monthly except for July and August. Regular subscription rate: $\$ 190.00$ a year (10 issues). Special rate: $\$ 95.00$ a year to individual members of supporting institutions.

Subscriptions, orders for numbers issued in the last three calendar years, and changes of address should be sent to Pacific Journal of Mathematics, P.O. Box 969, Carmel Valley, CA 93924, U.S.A. Old back numbers obtainable from Kraus Periodicals Co., Route 100, Millwood, NY 10546.

The Pacific Journal of Mathematics at P.O. Box 969, Carmel Valley, CA 93924 (ISSN 0030-8730) is published monthly except for July and August. Second-class postage paid at Carmel Valley, California 93924, and additional mailing offices. Postmaster: send address changes to Pacific Journal of Mathematics, P.O. Box 969, Carmel Valley, CA 93924.

PUBLISHED BY PACIFIC JOURNAL OF MATHEMATICS, A NON-PROFIT CORPORATION Copyright (C) 1991 by Pacific Journal of Mathematics 


\section{Pacific Journal of Mathematics}

Vol. 149, No. $2 \quad$ June, 1991

Manuel Alfaro Garcia, Mark Conger and Kenneth Hodges, The structure

of singularities in $\Phi$-minimizing networks in $\mathbf{R}^{2}$

Werner Balser, Dependence of differential equations upon parameters in

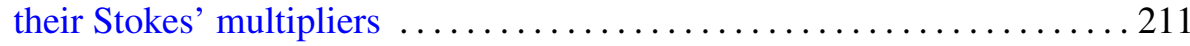

Enrico Casadio Tarabusi and Stefano Trapani, Envelopes of holomorphy

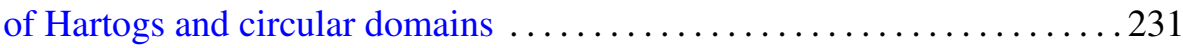

Hermann Flaschka and Luc Haine, Torus orbits in $G / P \ldots \ldots \ldots \ldots 251$

Gyo Taek Jin, The Cochran sequences of semi-boundary links .......... 293

Yasuyuki Kawahigashi, Cohomology of actions of discrete groups on

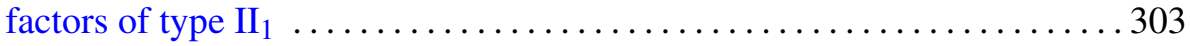

Ki Hyoung Ko and Lawrence Smolinsky, A combinatorial matrix in 3-manifold theory

W. B. Raymond Lickorish, Invariants for 3-manifolds from the combinatorics of the Jones polynomial

Peter Arnold Linnell, Zero divisors and group von Neumann algebras . . . . . 349

Bruce Harvey Wagner, Classification of essential commutants of abelian

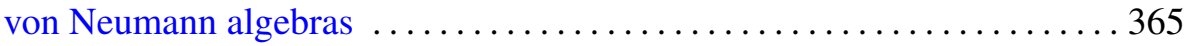

Herbert Walum, Multiplication formulae for periodic functions ..........383 